\title{
Enhancement of subsurface resistivity distribution mapping using MTEM dense reverse source array: A case study of Anambra basin, Nigeria.
}

\author{
Olalekan Fayemi, Qingyun Di, Ruo Wang \\ Adewole A. Ajala \\ Busayo O. Omisore \\ Key Laboratory of Shale Gas and Geoengineering Institute of Processing Engineering,CAS China University of Geosciences \\ Institute of Geology and Geophysics, CAS \\ Beijing, China \\ Beijing, China
} Beijing, China

\begin{abstract}
In this study, we presented a new multi transient electromagnetic (MTEM) data acquisition array to solve the challenges observed in using the conventional data acquisition setup in MTEM survey over complex geology. This involves the use of double transmitter dipoles and the recording of both the forward and reverse responses. The result obtained from using the dense reverse source (DRS) array to reconstruct the subsurface resistivity distribution over part of Anambra basin shows that the new method is more effective in mapping non horizontal hydrocarbon filled reservoir and very useful for general geophysical prospecting.
\end{abstract}

Keywords-MTEM; DRS array; Hydrocarbon reservoir; Anambra basin.

\section{INTRODUCTION}

Multi-transient electromagnetic method is a time domain controlled source electromagnetic method which uses transient current passed via grounded dipole electrodes to probe the subsurface geology. This method was first introduced by Wright et al. ${ }^{[7]}$ as a high resolution EM method for both onshore and offshore geophysical exploration study. MTEM application includes, 4D monitoring of $\mathrm{CO}_{2}$ sequestration process, mapping tar sand deposits, Gas hydrates identification, mineral exploration, deep groundwater exploration, nonconventional exploration, geothermal exploration et cetera ${ }^{[3-5]}$.

The conventional array for MTEM survey involves the use of single transmitter dipole and several receiver dipoles that are arranged collinear to the transmitter dipole, which is placed on one side of the profile (the red points in Fig. 1). Though, the results obtained from this array type gives a good representation of subsurface resistivity distribution, especially when dealing with near horizontal layer or horizontally laid and/or centralized targets, it is less efficient when dealing with a complex geological setup with the subsurface stratigraphy or target dipping at an angle greater than $40^{\circ}$ thereby leading to false interpretation of the position, geometry and dip of subsurface materials.

Also, Considering the fact that the impulse response recorded at any given transmitter-receiver dipole position is a cumulative response from the subsurface resistivity material(s) between the two dipoles, the accuracy of the obtained apparent resistivity section can be marred by the presence of artifacts or concealed targets which in turn can lead to erroneous interpretation.

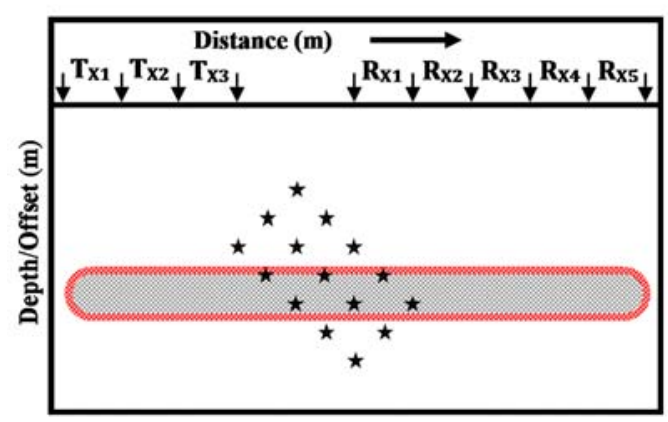

Fig. 1. Conventional MTEM survey array. The position of the receiver dipoles are kept fixed while the transmitter dipole is moved from left to right, that is, from Tx1 to Tx2, to Tx3 and so on.

In other to address the challenges faced when quick MTEM interpretation technique is used, we proposed the use of dense reverse source array for MTEM survey where complex and/or dipping subsurface material exists.

\section{Dense ReVErse Source ArRAy}

This method is based on the seismic refraction principle of on-shot and reverse shot, used in reconstructing true image of dipping layers. The transmitter dipole is placed at both ends of the multiple receivers, such that the receiver dipole measures the earth response from both directions (Fig. 2). This can also be achieved by moving a single transmitter all the way across the receiver layout in other to achieve the desired measurement of reverse transmission. The blue squares in fig. 2 represent the points and path of the reverse response, while the black asterisks represent the points and path of the conventional acquisition response.

This means that each midpoint will be surveyed at least twice. The two responses are processed separately in other to retrieve the earth impulse response from both directions, which are then used for quick estimation of subsurface apparent resistivity. The obtained apparent resistivities are combined for 1) quick accurate interpretation of subsurface resistivity distribution, 2) Removal of possible artifacts by 
visual inspection, and 3) Joint full waveform inversion for better estimation of the subsurface resistivity distribution and target's geometry.

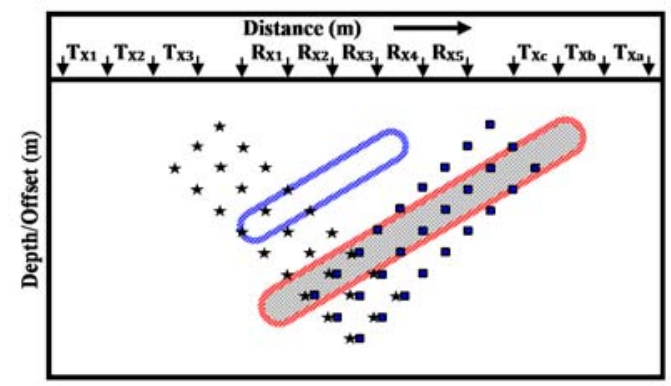

Fig. 2. Dense reverse source array. The position of the receiver dipoles are kept fixed while the transmitter dipoles placed at both ends are moved towards the receiver, that is, from Tx1 to Tx2, to Tx3 and from Txa to Txb, to Txc, until the current dipoles get to position Txo, which is the position of the current dipoles at minimum offset away from Rxm+1 and Rxm-1, respectively. Where Rxm is the position of the mid receiver dipole. The reverse data can be acquired while the conventional dipole source is being moved.

\section{CASE STUDY OF ANAMBRA BASIN}

The area of study is located within the Anambra basin of the lower Benue trough between latitude $6^{\circ} 00^{\circ} \mathrm{N}$ and $7^{\circ} 00^{\circ} \mathrm{N}$ of the equator and Longitude $6^{\circ} 30^{\circ} \mathrm{E}$ and $7^{\circ} 40^{\circ} \mathrm{E}$. The Anambra Basin which is located in the southwestern part of the Benue Trough has been identified as one of the major inland sedimentary basins in Nigeria. It is one of the three basins within The Federal Republic of Nigeria that has potential for both production and storage of hydrocarbon deposit. However, in comparison to the Niger Delta basin, little has been done to fully characterize this deposit. Most of the past and present works done in this area have been focused on source rock maturity and seismic exploration, with about 18 and less than 50 wells drilled in the entire basin ${ }^{[2]}$. Recent increase in pursuit of information necessary to optimize development in exploration and exploitation of petroleum in the Anambra Basin has led to further study on the basin. However, little or no direct electromagnetic survey has been carried out to complement seismic survey and minimize exploration risks and cost. Hence, in this study we proposed the use of Multitransient electromagnetic (MTEM) method as an onshore geophysical tool for oil exploration/prospecting over part of the Enugu arm of the Anambra basin, with focus on showing the efficiency and effectiveness of the dense reverse source array in relatively complex geological setup.

Based on information obtained from 4 oil wells and geothermal maturity section used in the prediction of probable oil producing region ${ }^{[1]}$, 2D resistivity model of the subsurface geological setup within the area of study was generated (Fig. 3 ). The model was built on a $75 \times 35$ grid space, using a grid spacing of $150 \mathrm{~m}$ along the $\mathrm{x}$-direction and $100 \mathrm{~m}$ along the $\mathrm{z}-$ direction. Using 2500 time steps and additional 15 cells for the PML region, the MTEM earth impulse response was modeled for offsets varying with respect to receiver position from 900 $\mathrm{m}$ to $6300 \mathrm{~m}$ at an interval of $300 \mathrm{~m}$.

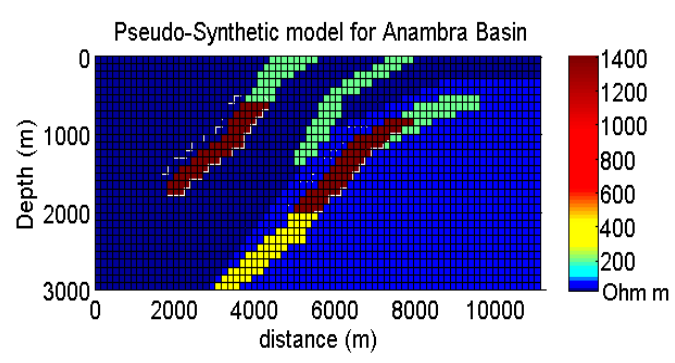

Fig. 3. 2D Pseudo-synthetic model for part of Anambra basin.

Using the conventional MTEM data acquisition setup, the transmitter dipole was initially placed at distance $500 \mathrm{~m}$ and then moved across the section. For quick data interpretation, the peak time of the modeled earth impulse response was used to calculate the subsurface apparent resistivity (Wilson, 1997). Fig. 4 shows the obtained $2 \mathrm{D}$ apparent resistivity plot on a common midpoint - offset section. The apparent resistivity distribution on the section indicates the presence of 2 distinct geo-electric mediums. The first medium is represented by yellow to red coloration, which reflects a resistive medium. The second medium in blue is the conductive surrounding medium. Though, the apparent resistivity section shows the existence of three dipping resistive materials as observed on the $2 \mathrm{D}$ model, the quality of the result is marred by the existence of an artifact that runs between distances $4000 \mathrm{~m}$ and $6200 \mathrm{~m}$. Also, the geometry of the resistive mediums are not well formed, especially mediums 2 and 3.

On the other hand, the apparent resistivity section obtained from the reverse survey (Fig. 5), when the new dense reverse source array was used, showed a better representation of both mediums 2 and 3. The apparent resistivity distribution on this section indicates the presence of 3 distinct geo-electric mediums. The first medium is represented by red coloration, which reflects a highly resistive medium between distances $5400 \mathrm{~m}$ and $7800 \mathrm{~m}$. This represents the Hydrocarbon filled region. The second medium is represented by light blue coloration between distances $5200 \mathrm{~m}$ and $7600 \mathrm{~m}$ at the top of the section and a steeply dipping structure at the far left. The last medium, which is majorly blue, is the conductive surrounding medium.

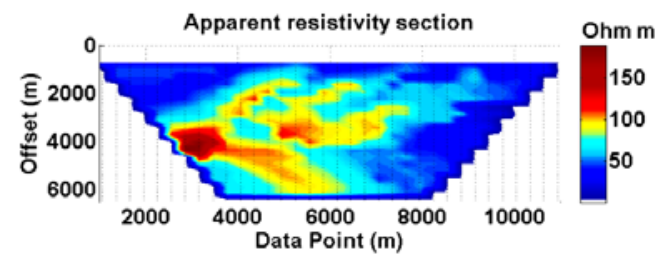

Fig. 4. 2D apparent resistivity section obtained from using the conventional MTEM data acquisition setup. 


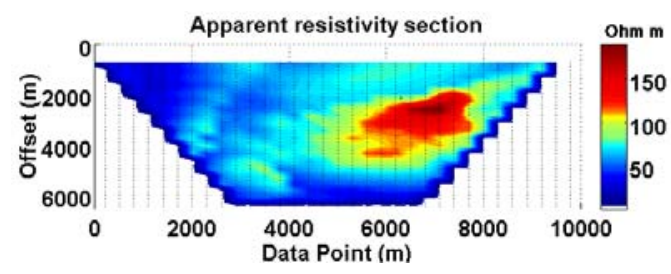

Fig. 5. 2D apparent resistivity section obtained from using the reverse survey.

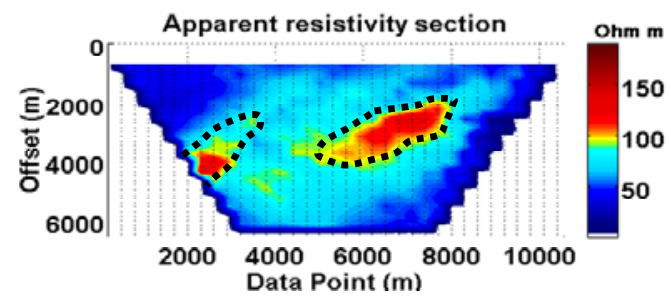

Fig. 6. Combined 2D apparent resistivity section obtained from the dense reverse source array.

The apparent resistivity distribution on the section indicates the presence of 2 distinct geo-electric mediums; the yellowish red to dark red coloration, which reflects a resistive to highly resistive reservoir formation and the deep blue color represents the conductive background medium.

Furthermore, the apparent resistivity values from the two sections were combined together by calculating the weighted average of the two values obtained from the earth impulse responses at each data point (current-receiver dipoles midpoint), using (1) below and were plotted in Fig. 6.

$$
R_{c}=\left[\left(R_{d} * w_{1}\right)+\left(R_{r} * w_{2}\right)\right] / 10
$$

where $R_{c}, R_{d}$, and $R_{r}$ are the combined apparent resistivity, direct source apparent resistivity and reverse source apparent resistivity, respectively. $w_{1}$ and $w_{2}$ represents the weight of the direct source apparent resistivity and reverse source apparent resistivity, respectively and their values range from 3 to 7 . Also, $w_{1}+w_{2}=10$. For this study, weighted ratio of 5:5 was used in calculating the $R_{c}$.

The apparent resistivity distribution on the section indicates the presence of 3 geo-electric mediums; the red coloration, which reflects a highly resistive hydrocarbon filled region. This runs between horizontal distances of about 1800 $\mathrm{m}$ to $3600 \mathrm{~m}$ and $5000 \mathrm{~m}$ to $7800 \mathrm{~m}$. The second medium is the conductive (deep Blue color) surrounding medium, while the third is characterized by indistinguishable light blue color. This represents in part the moderately resistive nonhydrocarbon filled reservoir. This runs from the surface to the bottom of the section. In comparison with the previous results, the dense reverse source array was very effective in mapping the hydrocarbon filled region. This shows that the new dense reverse source array is very effective in characterizing the subsurface resistivity distribution, especially in hydrocarbon exploration.

On the whole, the combined apparent resistivity section was in good agreement with the 2D Pseudo-synthetic model for part of Anambra basin, thus, showing the capability of the relatively new method as an effective geophysical tool to complement seismic data in hydrocarbon prospecting. Although, few discrepancies were observed (especially the inadequate mapping of the non-hydrocarbon filled reservoir), however the 2D modeling of MTEM earth response has been successful in the demonstration of good knowledge of the subsurface resistivity cross-section.

\section{CONCLUSION}

This paper successfully demonstrates the effectiveness of the dense reverse source array in mapping relatively complex subsurface geological composition. The study confirmed the validity of the method, as an effective tool for the detection of hydrocarbon within reservoir. With the new acquisition method, we were able to model the hydrocarbon bearing region effectively. Hence, it will be very efficient in both inland and marine study over the Anambra basin.

\section{ACKNOWLEDGMENT}

This research was supported by R\&D of Key Instruments and Technologies for Deep Resources Prospecting (the National R\&D Projects for Key Scientific Instruments), Grant No. ZDYZ2012-1-05-04. Also, the authors would like to thank the Third World Association of Sciences (TWAS) and the Chinese Academy of Sciences (CAS) for supporting the Ph.D. program with a fellowship.

\section{References}

[1] Ajala, A. A., 2012, Thermal maturity and burial history modeling of Anambra basin: Msc. Thesis, University of Ibadan, Nigeria.J. Clerk Maxwell, A Treatise on Electricity and Magnetism, 3rd ed., vol. 2. Oxford: Clarendon, 1892, pp.68-73.

[2] Akaegbobi, I. M., 2005, The Crabs eye-view of the organic sedimentological evolution of the Anambra Basin Nigeria: Hydrocarbon source potential and economic implications. University of Ibadan Press, Nigeria, $1-42$.

[3] Anderson, C., A. Long, A. Ziolkowski, B. Hobbs, and D. Wright, 2008. Multi-transient EM technology in practice, First Break, 26, 93-102.

[4] Engelmark, F., 2008. Improved Exploration, Appraisal and Production Monitoring with Multi-Transient EM Solutions. Back to Exploration 2008 CSPG CSEG CWLS Convention, 136 - 140.

[5] Fayemi, O. and Q. Y. Di, 2015. Application of MTEM method in deep ground water exploration studies. To be presented at the 12th China International Geo-electromagnetic induction workshop, Changsha, Hunan province, China. Oct 21 to Oct 23, 2015.

[6] Wilson, A. J. S., 1997, The equivalent wavefield concept in multichannel transient electromagnetic surveying: Ph.D. thesis, University of Edinburgh.

[7] Wright, D. A., A. Ziolkowski, and B. A. Hobbs, 2005. Detection of Subsurface Resistivity Contrasts With Application To Location Of fluids: U. S. Patent 6914433. 